\title{
Improved Smart Microgel Carriers for Catalytic Silver Nanoparticles
}

\author{
Timo Brändel, Viktor Sabadasch, Yvonne Hannappel, ${ }^{\odot}$ and Thomas Hellweg*(i) \\ Department of Physical and Biophysical Chemistry, Bielefeld University, Universitätsstrasse 25, 33615 Bielefeld, Germany
}

\section{Supporting Information}

\begin{abstract}
Acrylamide-based, thermoresponsive coreshell microgels with a linear phase transition region are used as improved carriers for catalytically active silver nanoparticles in the present study. In this context, we investigated the swelling behavior of the carriers and the stability of the silver nanoparticles inside the polymer network with photon correlation spectroscopy, transmission electron microscopy,

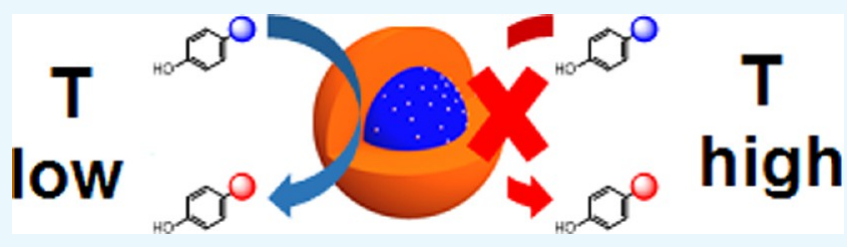
and by following the surface plasmon resonance of the nanoparticles. Depending on the cross-linker content of the microgel core, we observed very good stability of the nanoparticles inside the microgel network, with nearly no bleeding or aggregation of the nanoparticles over several weeks for core cross-linker contents of 5 and $10 \mathrm{~mol} \%$. The architecture of the hybrid particles in the swollen state was investigated with cryogenic transmission electron microscopy. The particles exhibit a core-shell structure, with the silver nanoparticles located mainly at the interface between the core and shell. This architecture was not used before and seems to grant advanced stability to the nanoparticles inside the network in combination with good switchability of the catalytic activity. This was measured by following the reduction of 4-nitrophenole, which is a well-studied model reaction. The obtained Arrhenius plots show that similar to previous works, the swelling of the core and shell can influence the catalytic activity of the silver nanoparticles. As mentioned before, the cross-linker content of the core seems to be a very important parameter for the switchability of the catalytic activity. A higher cross-linker content of the core seems to be connected to a stronger influence of the carrier swelling degree on the catalytic activity of the silver nanoparticles.
\end{abstract}

\section{INTRODUCTION}

During the last decade, hybrid materials combining the properties of metal nanoparticles and soft matter templates or carriers have become one of the most studied topics in different fields of science. ${ }^{1}$ The applications of these hybrid systems range from magnetic resonance imaging ${ }^{2-4}$ to plasmonics, $^{5-9}$ emulsifiers, ${ }^{10,11}$ drug targeting, ${ }^{12-15}$ medical applications, ${ }^{16}$ and catalysis. ${ }^{17-24} \mathrm{~A}$ very interesting class of catalytically active hybrid systems is silver nanoparticles embedded in stimuli-responsive microgels because silver nanoparticles are not only outstanding catalysts ${ }^{25-27}$ but also considered to be antimicrobial. ${ }^{28}$ Moreover, the polymer carrier prevents the silver nanoparticles from aggregating, and the use of a stimuli-responsive support may grant the possibility to tune the catalytic activity of the nanoparticles by triggering the respective stimulus in the microgel. ${ }^{29}$

In this study, thermoresponsive microgels are used as carriers for the silver nanoparticles. At a certain temperature, the so-called volume phase transition temperature (VPTT), the particles undergo a remarkable change in size due to the release of solvent. ${ }^{30,31}$ The most popular examples for thermoresponsive microgels are based on $\mathrm{N}$-isopropylacrylamide (NIPAM), ${ }^{31-33}$ but microgels prepared from $\mathrm{N}$ isopropylmethacrylamide (NIPMAM) have generated considerable attention as well. ${ }^{34,35}$ In fact, another very interesting class of microgels is based on the monomer N-npropylacrylamide (NNPAM), which leads to particles with a very steep phase transition at a rather low VPTT of $22^{\circ} \mathrm{C} .{ }^{36}$ The chemical composition of the microgel is not the only point that has to be considered. The particle architecture also plays an important role in the properties of microgels. ${ }^{37}$ Zeiser et al. ${ }^{38}$ and Berndt et al. ${ }^{39}$ showed that core-shell microgels, with different VPTTs in the core and in the shell, may exhibit a linear phase transition region between the respective VPTTs of the homopolymer microgels, and additionally the concentration of the cross-linker $N, N^{\prime}$-methylenebisacrylamide (BIS) has an important influence on the particle morphology and the microgel properties. ${ }^{40}$ Also, Jones et al. ${ }^{37}$ showed that coreshell microgels based on PNIPAM-co-AAc cores and PNIPAM shells show a different swelling behavior than simple copolymer microgels. The present study focuses on the mentioned core-shell approach as carriers for silver nanoparticles using different acrylamides compared to previous hybrid materials. The catalytic activity is followed exploiting the reduction of $p$-nitrophenole as a model reaction. We use core-shell microgels with different VPTTs of core and shell as templates to achieve two objectives. First, the stability of the nanoparticles inside the carrier particles should be improved and bleeding of the silver nanoparticles should be prevented even over several weeks. This point is very important because the long-term stability of the nanoparticles inside the microgel network ensures the reusability of the hybrid systems as a catalyst over several cycles. The second aim is to use the different temperature responses of the core and shell to

Received: December 14, 2018

Accepted: February 12, 2019

Published: March 4, 2019 
enhance the switchability of the catalytic activity. By adjusting the temperature above or below the VPTT of the shell, we expect an additional contribution to the catalytic activity of the silver nanoparticles, which could not be realized in templates with only one temperature response. ${ }^{29}$ A variation of the VPTT of the core also offers the possibility to adjust the swelling behavior of the microgel template and will therefore allow to tailor the response of the carriers in a very specific fashion.

To achieve the mentioned objectives, it is important that the nanoparticles are located mainly in the core part of the microgel. In our present study, we try to achieve a preferred localization of the nanoparticles by following the concept of Zhang et al., ${ }^{41}$ where carboxylic groups inside the microgel particles serve as a complex forming groups for added silver ions, which are subsequently reduced to silver nanoparticles. After the reduction, these groups may also act as anchors for the nanoparticles. The introduction of carboxylic groups is done by copolymerization of the core base polymer with acrylic acid (AAc). Since high amounts of acrylic acid not only influence the volume phase transition of microgels but also affect the structure of the microgels toward a lower network density, especially on the edges of the microgel particles, ${ }^{42,43}$ we use $5 \mathrm{~mol} \%$ acrylic acid as a comonomer in the core synthesis. Therefore, the hybrid systems will have an architecture that is schematically presented in Figure 1 after the synthesis of the silver nanoparticles.

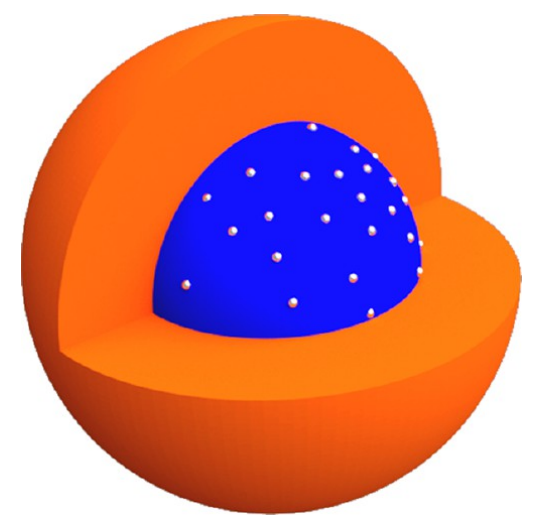

Figure 1. Schematic representation of the targeted architecture of the core-shell microgel/silver nanoparticle hybrids. The silver nanoparticles are located primarily inside the microgel core (blue), whereas the shell (orange) should improve the nanoparticle stability and switchability of the hybrid systems.

To develop a full set of different catalysts, we investigate the influence of two different parameters on the capability of core-shell microgels to serve as silver nanoparticle carriers. First, we varied the monomer that was used as the main core monomer between NIPAM, NIPMAM, and NNPAM, whereas the shell always consisted of the low-VPTT polymer PNNPAM; second, we varied the cross-linker content of the microgel core from 5 to $15 \mathrm{~mol} \%$, whereas the cross-linker content of the shell was kept at $5 \mathrm{~mol} \%$. The core-shell microgel templates are characterized by photon correlation spectroscopy (PCS), scanning electron microscopy, and atomic force microscopy (AFM). The hybrid systems with silver nanoparticles are characterized by different transmission electron microscopy (TEM) techniques, including cryogenic TEM (cryo-TEM), and by following the plasmon resonance of the silver nanoparticles to investigate the stability and size of the nanoparticles. Finally, the catalytic activity will be investigated at different temperatures and related to the swelling of the microgel template.

\section{RESULTS AND DISCUSSION}

2.1. Microgel Characterization. We successfully synthesized the microgel particles listed in Table 1 with their respective sample compositions.

After the synthesis of all core-shell microgels, the particles were investigated with atomic force microscopy (AFM). Figure 2 shows three representative images of the microgels in the dry state.

In Figure 2, all images show particles with a circular cross section. The radius of the particles was analyzed with free software ImageJ and depends strongly on the core material. While the particles PNI5@PNN have an average radius of 275 $\mathrm{nm}$, the particles PMAM5@PNN are much bigger with an average radius of $440 \mathrm{~nm}$. The smallest particles are the PNN5@PNN core-shell microgels with an average radius of $160 \mathrm{~nm}$. These size differences are caused by the use of sodium dodecyl sulfate (SDS) during the core synthesis. Wedel et al. ${ }^{44}$ and von Nessen et al. ${ }^{34}$ discussed the different influences of SDS on the size of acrylamide-based microgels earlier. In principle, SDS usage during the synthesis has a strong influence on the size of PNNPAM microgels; the influence on PNIPAM microgels is rather moderate and for PNIPMAM microgels the influence of the surfactant is smaller.

Additionally, we investigated the swelling behavior of all core-shell microgels with photon correlation spectroscopy (PCS). The obtained swelling curves are shown in Figure 3.

The swelling curves in Figure $3 \mathrm{a}, \mathrm{b}$ are typical examples of the swelling behavior of core-shell microgels composed of core microgels with a high volume phase transition temperature (VPTT) and shell material, which exhibits a low VPTT. According to the work by Zeiser et al., ${ }^{38}$ two main phase

Table 1. Sample Composition and Sample Names for all Core-Shell Microgels, which Were Investigated in the Present Study

\begin{tabular}{ll} 
sample name & \multicolumn{1}{c}{ core composition } \\
PNI5@PNN & NIPAM-co-AAc 5 mol \% BIS \\
PNI10@PNN & NIPAM-co-AAc $10 \mathrm{~mol} \% \mathrm{BIS}$ \\
PNI15@PNN & NIPAM-co-AAc $15 \mathrm{~mol} \% \mathrm{BIS}$ \\
PMAM5@PNN & NIPMAM-co-AAc $5 \mathrm{~mol} \% \mathrm{BIS}$ \\
PMAM10@PNN & NIPMAM-co-AAc $10 \mathrm{~mol} \% \mathrm{BIS}$ \\
PMAM15@PNN & NIPMAM-co-AAc $15 \mathrm{~mol} \% \mathrm{BIS}$ \\
PNN5@PNN & NNPAM-co-AAc $5 \mathrm{~mol} \% \mathrm{BIS}$ \\
PNN10@PNN & NNPAM-co-AAc $10 \mathrm{~mol} \% \mathrm{BIS}$ \\
PNN15@PNN & NNPAM-co-AAc $15 \mathrm{~mol} \% \mathrm{BIS}$
\end{tabular}

shell composition

NNPAM $5 \mathrm{~mol} \%$ BIS

NNPAM $5 \mathrm{~mol} \%$ BIS

NNPAM $5 \mathrm{~mol} \%$ BIS

NNPAM 5 mol \% BIS

NNPAM 5 mol \% BIS

NNPAM 5 mol \% BIS

NNPAM $5 \mathrm{~mol} \%$ BIS

NNPAM 5 mol \% BIS

NNPAM 5 mol \% BIS 

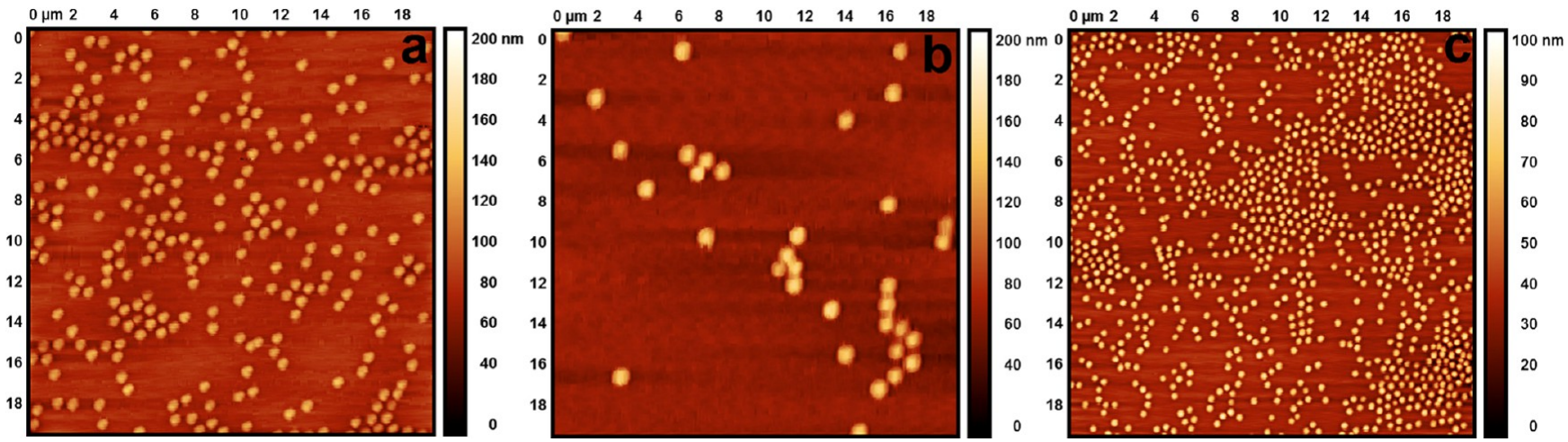

Figure 2. Representative AFM images of three different core-shell microgels PNI5@PNN (a), PMAM5@PNN (b), and PNN5@PNN (c) spincoated on a silicon wafer. The measurements were performed in semicontact mode in the dry state.
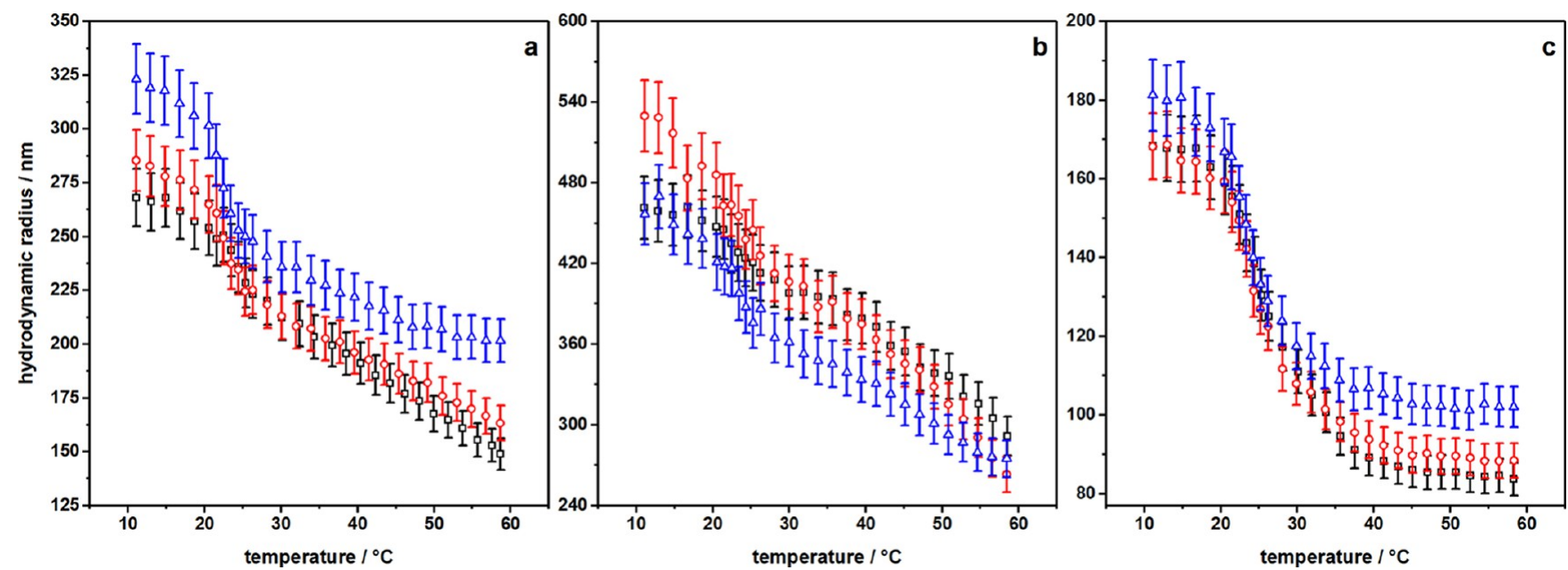

Figure 3. PCS swelling curves of all core-shell microgel templates with core cross-linker contents of 5 mol \% (black squares), 10 mol \% (red circles), and $15 \mathrm{~mol} \%$ (blue triangles). The microgel composition varies from PNIPAM-co-AAc@PNNPAM (a) over PNIPMAM-co-AAc@ PNNPAM (b) to PNNPAM-co-AAc@PNNPAM (c).

transition regions can be identified. At ca. $22{ }^{\circ} \mathrm{C}$, which corresponds to the VPTT of PNNPAM, a steep decrease in the hydrodynamic radius is observable. This corresponds to the deswelling process of the shell. After that, a large linear phase transition region is observable. In our case, the broad linear region is probably caused by an interpenetration of the shell inside the core-corona structure of the acrylamide-acrylic acid copolymer microgel cores. ${ }^{37,43}$ This assumption is strongly supported by the fact that the shell size directly depends on the cross-linker content of the core for all PNIPAM-co-AAc@ PNNPAM microgels. The higher the cross-linker content of the core, the bigger the PNNPAM shell, which is due to a limited penetration depth of the PNNPAM inside the core microgel. Interestingly, the fully collapsed state of the microgel is only reached for the PNI15@PNN core-shell microgel. We suppose that the reason for this is that the charge density inside the microgel particles is the lowest because of the high cross-linker content. Another important point is that the crosslinker content of the core influences the slope of the linear phase transition region in a way that the slope decreases with increasing cross-linker content. This effect was already described by Zeiser et al. ${ }^{38}$ and can be attributed to a "corsett" effect. This term was introduced by Berndt et al. ${ }^{39}$

However, for the PNIPMAM-co-AAc@PNNPAM microgels, the cross-linker dependency of the shell size is not observable. At this point, it is to be mentioned that the scattering intensity of the PNIPMAM-based core-shell microgels was very low at temperatures between 10 and $22{ }^{\circ} \mathrm{C}$ because the measured angle of $60^{\circ}$ is very close to the form factor minimum of the particles with a radius of 450-500 $\mathrm{nm}$. Therefore, the determination of the hydrodynamic radius is not very accurate at these temperatures. However, the cross-linker content dependency of the slope of the linear phase transition region is also observable for the PNIPMAM-co-AAc@PNNPAM microgels. In the case of the PNIPMAM-based core-shell microgels, the fully collapsed state is not reached in the investigated temperature range, which is due to the high VPTT of the core microgels.

Interestingly, the swelling curves of all PNNPAM-co-AAc@ PNNPAM microgels show a clearly different behavior than the other core-shell microgels. In this case, the size of the shell seems to be less pronounced compared to the PNIPAM-coAAc@PNNPAM microgels and also no dependence of the core cross-linker content is observable for the volume phase transition. For all PNNPAM-co-AAc@PNNPAM microgels, the swelling behavior is clearly dominated by the deswelling process of the shell at approximately $22{ }^{\circ} \mathrm{C}$. The influence of the core transition is only observable in a broadening of the volume phase transition in general. But all in all, no significant change in the swelling behavior is observable between all different PNNPAM-co-AAc@PNNPAM core-shell microgels with changing BIS contents. Finally, it should be mentioned 
that due to the lower VPTT of PNNPAM, the fully collapsed state is reached for all core cross-linker contents in this case.

To summarize the previous part, a strong dependency of the swelling curves on the core material of the microgels was observed. While PNIPAM-co-AAc@PNNPAM and PNIPMAM-co-AAc@PNNPAM core-shell microgels show a linear phase transition region, PNNPAM-co-AAc@PNNPAM microgels do not exhibit a comparable swelling behavior. We suppose that the appearance of the mentioned linear region is not only determined by a difference in the VPTT, but also strongly influenced by the particle architecture, which seems to be different if the core material is varied between the acrylamides. This can be supported by the findings of Jones et al., ${ }^{37}$ who did not observe a linear phase transition for coreshell microgels with a PNIPAM-co-AAc core and a PNIPAM shell. They found a two-step phase transition for those microgels. However, the differences in the swelling behavior are not necessarily transferable and/or problematic for the use of the core-shell microgels as smart carriers for silver nanoparticles. This subject is addressed in the following section.

2.2. Characterization of Microgel/Nanoparticle Hybrids. 2.2.1. PNIPAM-CO-AAc@PNNPAM Microgels. After the synthesis of the microgel/nanoparticle hybrids, we performed cryogenic transmission electron microscopy (cryo-TEM) measurements on the system PNI10@PNN to check if the targeted particle architecture could be realized. To investigate the swollen state of the core and shell, the temperature was adjusted to $20^{\circ} \mathrm{C}$. The cryo-TEM image is displayed in Figure 4.

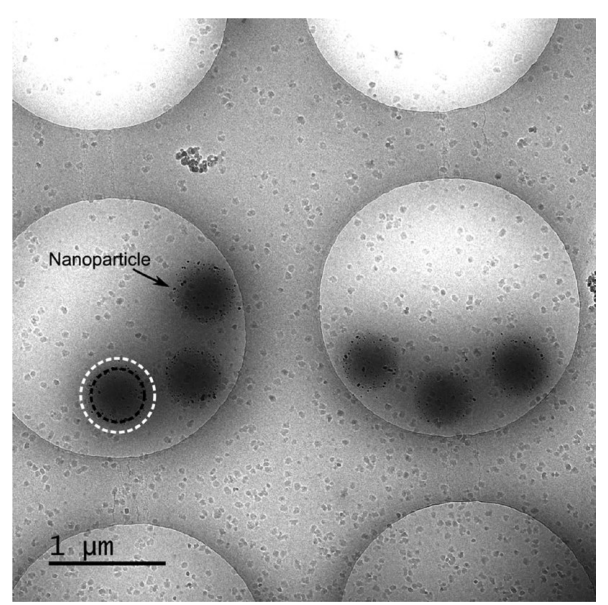

Figure 4. Cryo-transmission electron microscopy image of PNI10@ PNN decorated with silver nanoparticles. The preparation of the sample was performed at $20{ }^{\circ} \mathrm{C}$ to display the swollen state of the core-shell microgel/silver nanoparticle hybrids. The dashed circles indicate the microgel core (black) and the microgel shell (white).

The cryo-TEM image in Figure 4 shows that the particles in the swollen state retain their circular shapes, unaffected by the decoration with silver nanoparticles. The nanoparticle formation was successful, and the nanoparticles seem to be located on the interface between the microgel core, which is clearly visible with a high contrast, and the microgel shell, which is represented by the light gray corona around the microgel cores. The location of the nanoparticles at the interface between the core and shell is actually expected if the structural properties of the microgel support are taken into account. It was shown that PNIPAM-co-AAc microgels, which were synthesized via the classical procedure introduced by Pelton, have the carboxylic functional groups located mainly in the outer region of the microgel particle. ${ }^{43,45}$ Consequently, if an interpenetration between the PNNPAM shell and the PNIPAM-co-AAc core would occur, the carboxylic groups would be located at the interface between the core and shell. Since these carboxylic groups are intended to favor the formation of silver nanoparticles inside the microgel network, the nanoparticles are also formed at the interface between the core and shell. The high density caused by the interpenetration in combination with the electrostatic interaction with the carboxylic groups fixes the nanoparticles in the respective regions during and after their formation. Obviously, the silver nanoparticle/core-shell microgel hybrids exhibit the intended structure, which is presented as a concept in Figure 1. This makes them interesting candidates for the measurement of catalytic activity of the nanoparticles, which is presented in Section 2.3.

To ensure the success of the silver nanoparticle synthesis for the other PNIPAM-co-AAc@PNNPAM-based hybrid materials, we performed TEM measurements in the dry state for all samples. The resulting TEM images are shown in Figure 5.

The TEM images in Figure 5 show that the nanoparticle synthesis was successful for all PNIPAM-co-AAc@PNNPAM microgels. It has to be mentioned that the PNNPAM shell is not visible in the TEM measurements because of the low contrast of the very thin polymer shell in the fully collapsed and dried state. Furthermore, the cross-linker content of the core seems to have an influence on the stability of the silver nanoparticles inside the core-shell microgels. In the case of PNI5@PNN (a), a varying nanoparticle load of the three microgels is observable, but no nanoparticles on the outside of the microgel particles are visible. When the core cross-linker content increases to PNI10@PNN (b), the nanoparticle load is nearly constant, but in this case, some of the nanoparticles seem to bleed from the network during the drying process of the microgel on the TEM grid. In contrast to the dry TEM images, there are no nanoparticles outside of the microgels in Figure 4, but some nanoparticles seem to be located inside the shell and not at the core-shell interface. We suppose that these nanoparticles, which are not located in the interfacial region of the core-shell microgels, get detached during the drying process. The particles with the highest cross-linker content of the core PNI15@PNN (c) seem to exhibit a drastically increased nanoparticle load, but here also a very strong bleeding of the nanoparticles during the drying process can be observed. Additionally, the aggregation tendency of the silver nanoparticles is significantly higher in the last TEM image (c) than in the previous ones. Hence, it seems straightforward to conclude that the nanoparticle stability inside the microgel network decreases with increasing crosslinker content of the core. The number of nanoparticles per microgel and the mean size of the silver nanoparticles were analyzed for all samples. The information is given in the Supporting Information (SI).

Since bleeding of the silver nanoparticles occurred during the drying process, it is necessary to investigate the nanoparticle stability inside the microgel network in solution. Because of that, we analyzed the surface plasmon resonance of the microgel/silver hybrids over one month in a time interval of 2 weeks. The results are presented in Figure 6. 


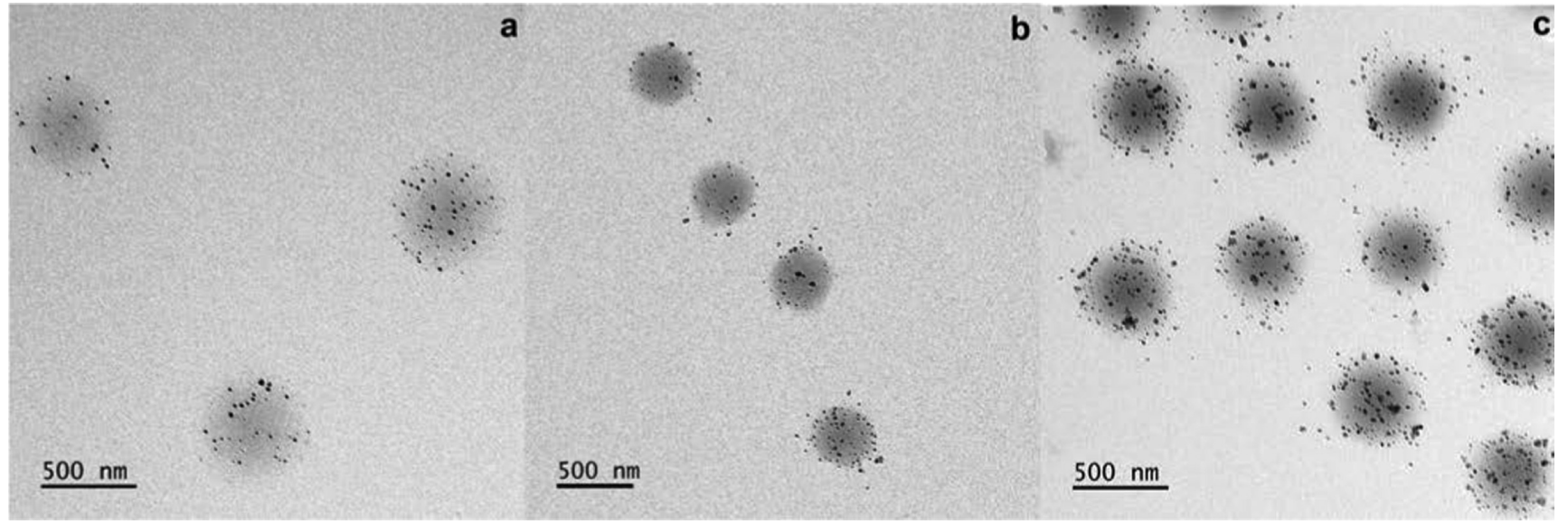

Figure 5. Transmission electron microscopy images of PNIPAM-co-AAc@PNNPAM core-shell microgel/silver nanoparticle hybrid systems in the dry state. The cross-linker content of the PNIPAM-co-AAc core varies from $5 \mathrm{~mol} \%$ (a) over $10 \mathrm{~mol} \%$ (b) to $15 \mathrm{~mol} \%$ (c) BIS with respect to the thermoresponsive material.

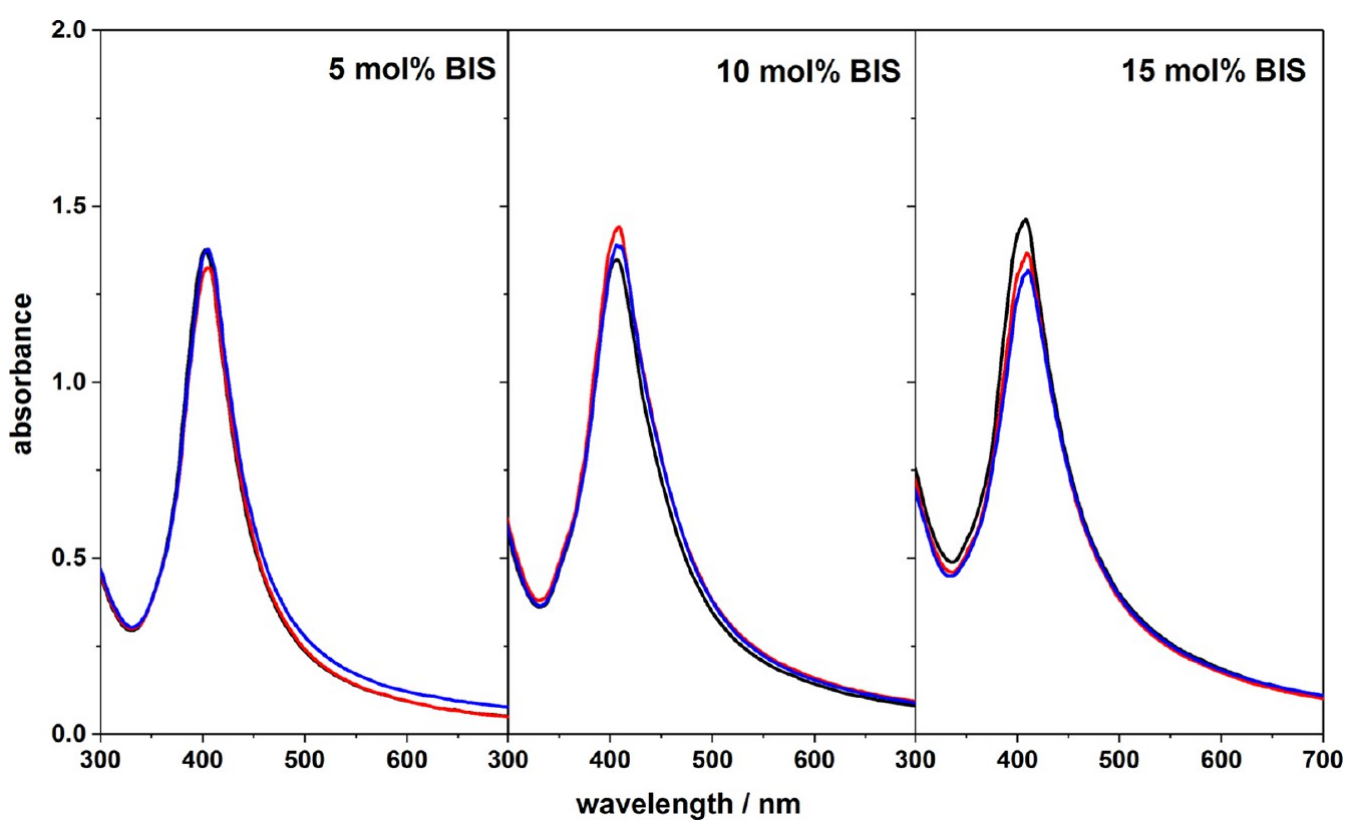

Figure 6. Surface plasmon resonance measurements of silver nanoparticles inside PNIPAM-co-AAc@PNNPAM microgels. The measurements were performed directly after the purification of the hybrid systems (black curves) and after storage times of 14 days (red curves) and 28 days (blue curves). The samples were stored at room temperature.

All samples in Figure 6 show the typical plasmon resonance band of silver nanoparticles at around $400 \mathrm{~nm}$, which corresponds to a mean size of $15 \mathrm{~nm}$. For the samples based on PNI5@PNN and PNI10@PNN, no change in the peak position or overall intensity is observable within the error of the measurements, but the hybrid system based on PNI15@ PNN shows substantial aging effects as expected based on the TEM measurements. The higher nanoparticle load directly after the purification of the core-shell microgels results in a higher absorbance, but the overall intensity of the plasmon resonance decreases over time, which corresponds to the bleeding of the material, and the peak is shifted toward higher wavelengths, which corresponds to aggregation of small silver nanoparticles. From both observations, the TEM measurements and the plasmon resonance measurements, we conclude that for the PNIPAM-co-AAc@PNNPAM core-shell microgels, only the samples with 5 and $10 \mathrm{~mol} \%$ cross-linker contents in the core are suitable templates for silver nanoparticles. If the core cross-linker content is increased further to $15 \mathrm{~mol} \%$, the silver nanoparticles are not stabilized inside the microgel network very well and therefore tend to aggregate and bleed. Consequently, an advanced switchability of the catalytic activity is only to expect for the hybrid systems based on PNI5@PNN and PNI10@PNN.

2.2.2. PNIPMAM-CO-AAc@PNNPAM Microgels. Also for the PNIPMAM-co-AAc@PNNPAM-based hybrid materials, TEM measurements were performed in the dry state. This was done to show the success of the nanoparticle synthesis and to study the influence of the cross-linker content of the core. The results are shown in Figure 7.

In Figure 7 mostly the same trends that were found for the PNIPAM-co-AAc@PNNPAM-based hybrids are observable. The nanoparticle synthesis was successful for all three core cross-linker contents, but the nanoparticle stability and the grade of the nanoparticle load vary between the different microgels. In general, the nanoparticle load of the PNIPMAM- 


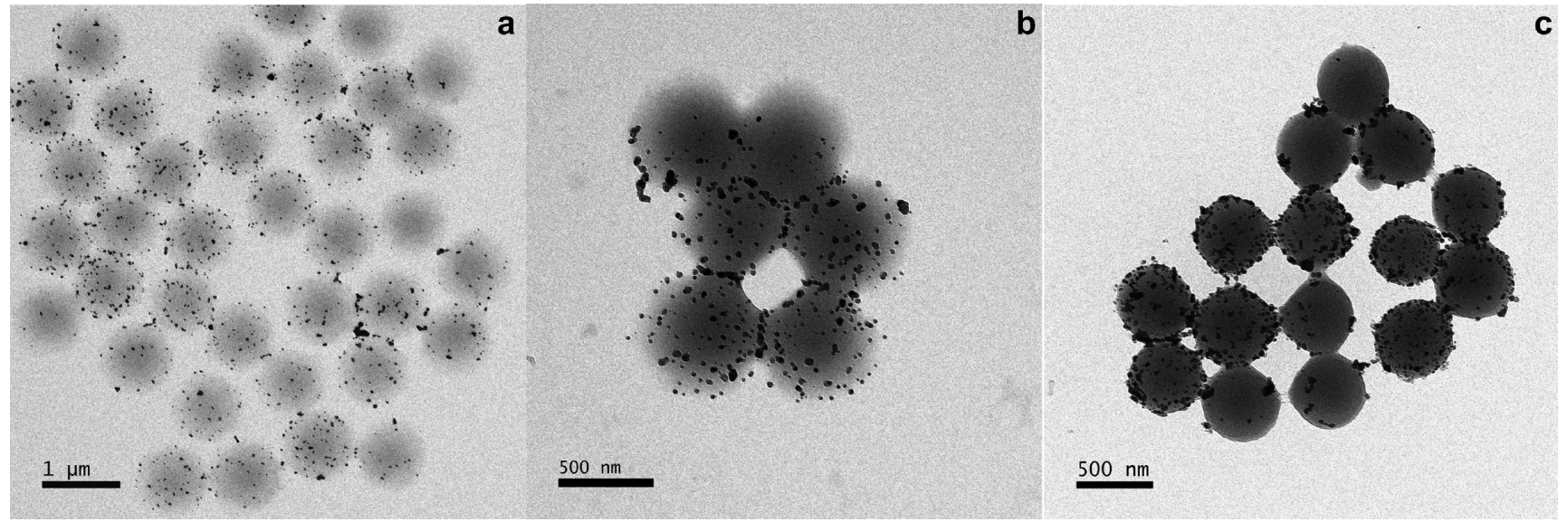

Figure 7. Transmission electron microscopy images of PNIPMAM-co-AAc@PNNPAM core-shell microgel/silver nanoparticle hybrid systems in the dry state. The cross-linker content of the PNIPMAM-co-AAc core varies from $5 \mathrm{~mol} \%$ (a) over $10 \mathrm{~mol} \%$ (b) to $15 \mathrm{~mol} \%$ (c) BIS with respect to the thermoresponsive material.

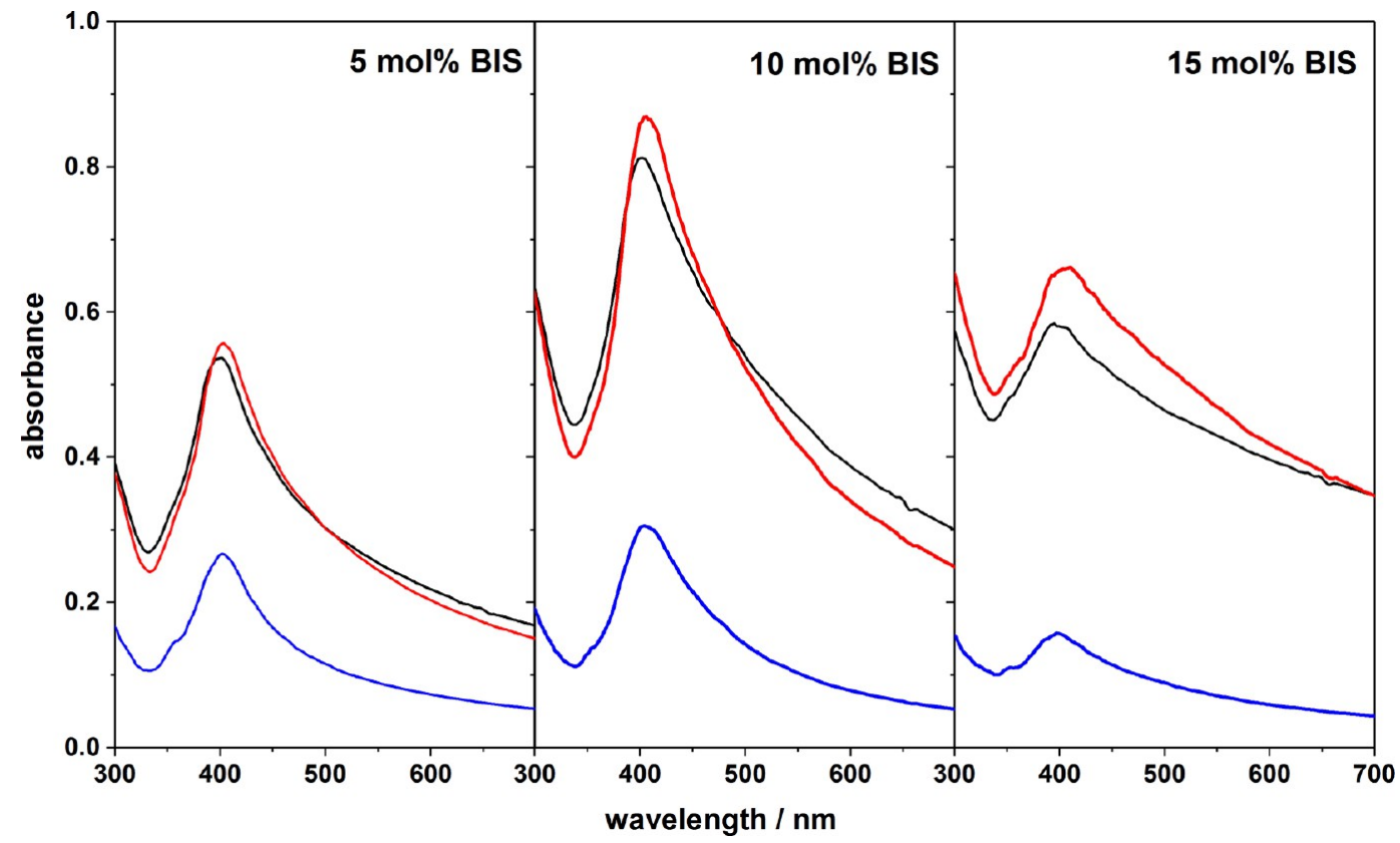

Figure 8. Surface plasmon resonance measurements of silver nanoparticles inside PNIPMAM-co-AAc@PNNPAM microgels. The measurements were performed directly after the purification of the hybrid systems (black curves) and after storage times of 14 days (red curves) and 28 days (blue curves). The samples were stored at room temperature.

co-AAc@PNNPAM microgels seems to be higher compared to the PNIPAM-co-AAc@PNNPAM-based hybrid systems. This increase could be caused by the increase in size of the template nanoparticles. However, the growing number of nanoparticles seems to be associated with an increase of the polydispersity. Additionally, a lot more aggregates are observable in the TEM images in Figure 7 compared to Figure 5. These observations can be verified when the mean radius of the silver nanoparticles in all TEM images is analyzed (see SI). In this case, especially the systems with $10 \mathrm{~mol} \%$ BIS and $15 \mathrm{~mol} \%$ BIS in the core show an increased tendency to bleed out during the drying process. Here, many nanoparticles are observable, which lay at the interface between adjacent core-shell microgels. Again in the TEM measurements, only the core-shell microgels with 5 and $10 \mathrm{~mol} \%$ BIS in the core seem to be suitable catalyst carriers as the nanoparticles are only distributed on the edges of the core-shell microgels in the case of $15 \mathrm{~mol} \%$ BIS in the core. Hence, this trend is preserved for both types of monomers. To investigate the silver nanoparticle stability inside the microgel network in the swollen state, also surface plasmon resonance measurements were performed for the PNIPMAM-co-AAc@PNNPAM-based hybrid systems and are shown in Figure 8.

Figure 8 shows that the surface plasmon resonance of the silver nanoparticles incorporated in the PNIPMAM-co-AAc@ PNNPAM microgels differs significantly from the surface plasmon resonance in the PNIPAM-co-AAc-based core-shell microgels (see Figure 6). Most prominently, an enormous decrease in the absorbance is observable after a storage time of 28 days. This is connected not only to an increased bleeding of the silver nanoparticles out of the polymer network, but also to aggregation phenomena of the microgels themselves since the scattering background of the microgels is also reduced drastically. The precipitation of large microgel aggregates can 
be observed by eye too, as the photograph in Figure 9 shows. Nevertheless, for shorter storage times, the surface plasmon

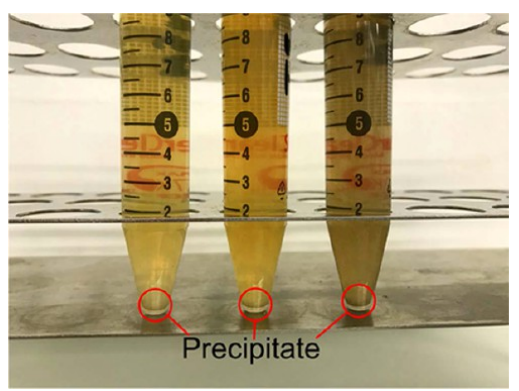

Figure 9. Photograph of PNIPMAM-co-AAc@PNNPAM microgels decorated with silver nanoparticles after a storage time of 28 days. The cross-linker content of the PNIPMAM-co-AAc core varies between $5 \mathrm{~mol} \%$ (left), $10 \mathrm{~mol} \%$ (middle), and $15 \mathrm{~mol} \%$ (right). The photograph was taken by Brändel.

resonance of the silver nanoparticles does not change significantly, especially for the samples based on PMAM5@ PNN and PMAM10@PNN. In the case of the hybrid system based on the core-shell microgels with $15 \mathrm{~mol} \%$ BIS in the core, a broadening of the plasmon resonance is observable. This is connected to an increased fraction of the nanoparticles in the water phase, which means the nanoparticles are likely to detach from the microgel surface. This observation is in line with the increased bleeding tendency of the silver nanoparticles in the TEM measurements. Therefore, it seems that comparable to the PNIPAM-co-AAc@PNNPAM-based coreshell microgels, a cross-linker content of $15 \mathrm{~mol} \%$ in the core is not suitable to design a carrier for silver nanoparticles.

In the photograph in Figure 9, some precipitated material is observable on the bottom of the samples. This precipitation is not only silver nanoparticles, which bled from the core-shell microgel hybrids, but seems to be composed of microgel hybrid systems too. This is supported by the fact that the precipitate can be redispersed very easily just by gently shaking the sample. Obviously, the decoration of the microgels with the silver nanoparticles seems to increase the aggregation tendency of the PNIPMAM-co-AAc@PNNPAM microgels. This can also be seen in the TEM measurements of the dried particles in Figure 7, where the nanoparticles form interconnections between the core-shell microgels. This different behavior compared to the PNIPAM-co-AAc@ PNNPAM-based hybrid materials could be caused by a change in the silver nanoparticle distribution inside of the polymer network.

2.2.3. PNNPAM-CO-AAC@PNNPAM Microgels. In the temperature-dependent PCS measurements in Figure 3, a different behavior of the PNNPAM-co-AAc@PNNPAM microgels was observed compared to the other core-shell microgels. To investigate if the PNNPAM-co-AAc@PNNPAM microgels also show a different capability for nanoparticle stabilization, we performed TEM measurements in the dry state. The results can be seen in Figure 10.

In contrast to the PCS swelling curves, a very similar behavior of the PNNPAM-co-AAc@PNNPAM microgel/ nanoparticle hybrids is observable. The synthesis of silver nanoparticles was successful. However, the nanoparticle load still varies between the microgel particles for all cross-linker contents, and the bleeding tendency still increases with increasing BIS content. Obviously, the cross-linker content of the microgel core plays the most important role concerning the stability of nanoparticles in the core-shell microgels as all microgels show the same tendency to lose nanoparticles when the cross-linker content is increased. This is a rather important fact because it means that the monomer composition of the core-shell microgels can easily be tailored toward many different conditions regarding the VPTT, length of the linear phase transition region, and size of the carrier particles without influencing the ability to stabilize the nanoparticles drastically. This makes the core-shell microgel/silver nanoparticle hybrids very flexible for use under many different application conditions.

Finally, surface plasmon resonance measurements were also performed for the PNNPAM-co-AAc-based hybrid materials to investigate the nanoparticle stability in the swollen state. The results are shown in Figure 11.

The surface plasmon resonance of the silver nanoparticles in Figure 11 seems to be rather comparable to the measurements that were performed for the PNIPAM-co-AAc@PNNPAM (see Figure 6)-based hybrid systems. The resonance band is slightly broadened compared to these measurements, but the general

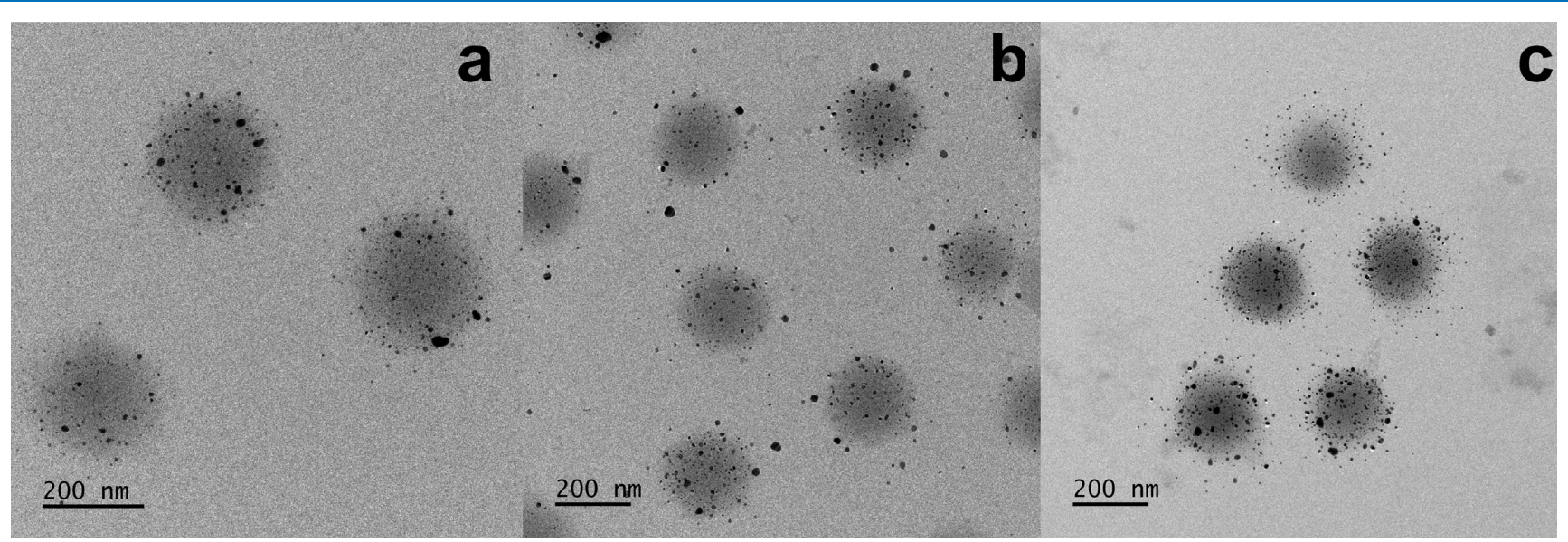

Figure 10. Transmission electron microscopy images of PNNPAM-co-AAc@PNNPAM core-shell microgel/silver nanoparticle hybrid systems in the dry state. The cross-linker content of the PNNPAM-co-AAc core varies from $5 \mathrm{~mol} \%$ (a) over $10 \mathrm{~mol} \%$ (b) to $15 \mathrm{~mol} \%$ (c) BIS with respect to the thermoresponsive material. 


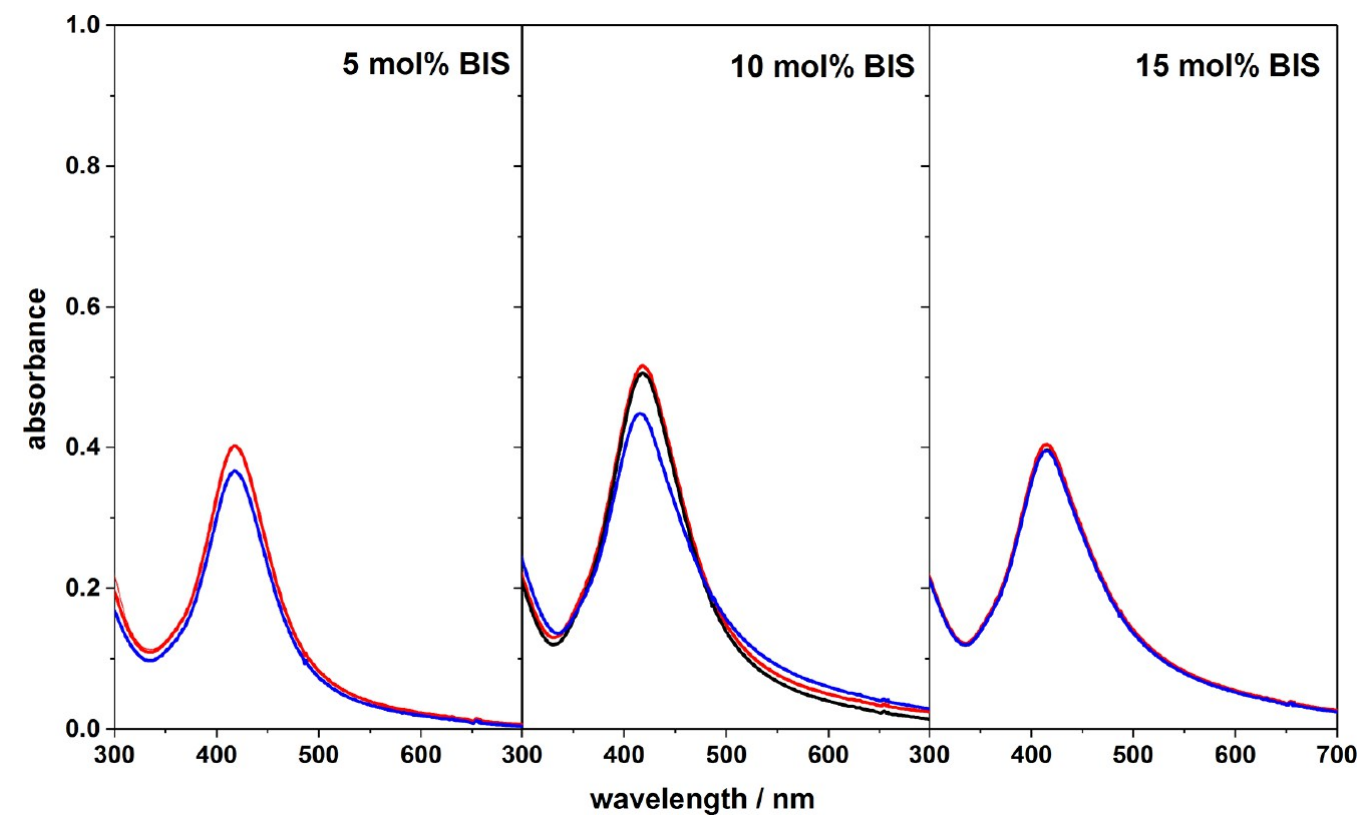

Figure 11. Surface plasmon resonance measurements of silver nanoparticles inside PNNPAM-co-AAc@PNNPAM microgels. The measurements were performed directly after the purification of the hybrid systems (black curves) and after storage times of 14 days (red curves) and 28 days (blue curves). The samples were stored at room temperature.

tendencies are preserved. There seems to be no significant bleeding for all microgels after 14 days, and only slight changes are observable for the measurements, which were performed after 28 days. In contrast to the PNIPMAM-based hybrid systems, no aggregation and flocculation of the particles can be observed. Therefore, we suggest that the colloidal stability of the hybrid systems based on PNNPAM-co-AAc is higher, which seems to be connected to a lower microgel particle size. However, in the case of the PNNPAM-co-AAc@PNNPAMbased hybrid systems, it seems that particles with all three cross-linker contents in the core microgel are appropriate to serve as nanoparticle carriers. No aging of the nanoparticles is observed, and the bleeding of the particles is nearly negligible. But in the TEM measurements, we found the same trends that were observed for all other microgels, and therefore we are going to concentrate on the analysis of the catalytic activity on the nanoparticles attached to the microgels PNN5@PNN and PNN10@PNN.

2.3. Catalytic Activity of Microgel/Nanoparticle Hybrids. In the previous section, it was shown that only core-shell microgels with a maximum core cross-linker content of $10 \mathrm{~mol} \%$ seem to be appropriate to be used as smart catalyst carriers. Therefore, we only investigated hybrid systems based on the samples PNI5@PNN, PNI10@PNN, PMAM5@PNN, PMAM10@PNN, PNN5@PNN, and PNN10@PNN with respect to their catalytic activity. As a model reaction, we used the reduction of 4-nitrophenole to 4aminophenole and followed the reaction by UV-vis spectroscopy. The reaction scheme is presented in Figure 12.

This well-known reaction is often exploited to study the influence of smart carrier systems on catalytic nanoparticles. ${ }^{24,46}$ Furthermore, Roa et al. ${ }^{47}$ and Angioletti et al. ${ }^{48}$ recently developed a model to describe the influence of the volume phase transition of PNIPAM on the catalytic activity of metal nanoparticles. In their work, they considered different architectures, which allow one to compare the results that were obtained in the present study with the theoretical model. In<smiles>Nc1ccc(O)cc1</smiles>

Figure 12. Reduction of 4-nitrophenole to 4-aminophenole is catalyzed by silver nanoparticles and can be used as a model reaction to investigate their catalytic activity.

principle, Roa et al. observed a decrease in the catalytic activity of the nanoparticles in the region of the VPTT of the polymer network, which can be explained by hindered diffusion of the reactants through the network because of the decreasing mesh size. Upon further increase of the temperature, the catalytic activity should increase again. This effect is caused by a change in the Gibbs free energy of solvation of the reactants inside the polymer network. From the TEM and especially the cryo-TEM measurements, the hybrid systems based on the samples PNI10@PNN, PMAM10@PNN, and PNN10@PNN seem to be the most promising candidates regarding an advanced switchability of the catalytic activity. Figure 13 shows the Arrhenius plots of these systems in comparison with the temperature-dependent hydrodynamic radius of the carrier systems. Systems with a passive carrier, for example, a spherical polyelectrolyte brush, would exhibit a fully linear relation of the reaction's logarithmic apparent rate constant and $1 / T{ }^{49}$ Deviations from the linear behavior are related to changes in the environment of the nanoparticles due to the response of the microgel network to the temperature.

Figure 13 shows that at least two types of core-shell microgels exhibit a deviation from the standard Arrhenius behavior. The difference shows a distinct intensity for each of the systems. For the hybrid systems based on the sample PNI10@PNN, we observe two deviations from the expected linear behavior. The first one is very small and is connected to the VPTT of the PNNPAM shell at around $22{ }^{\circ} \mathrm{C}$. The other 

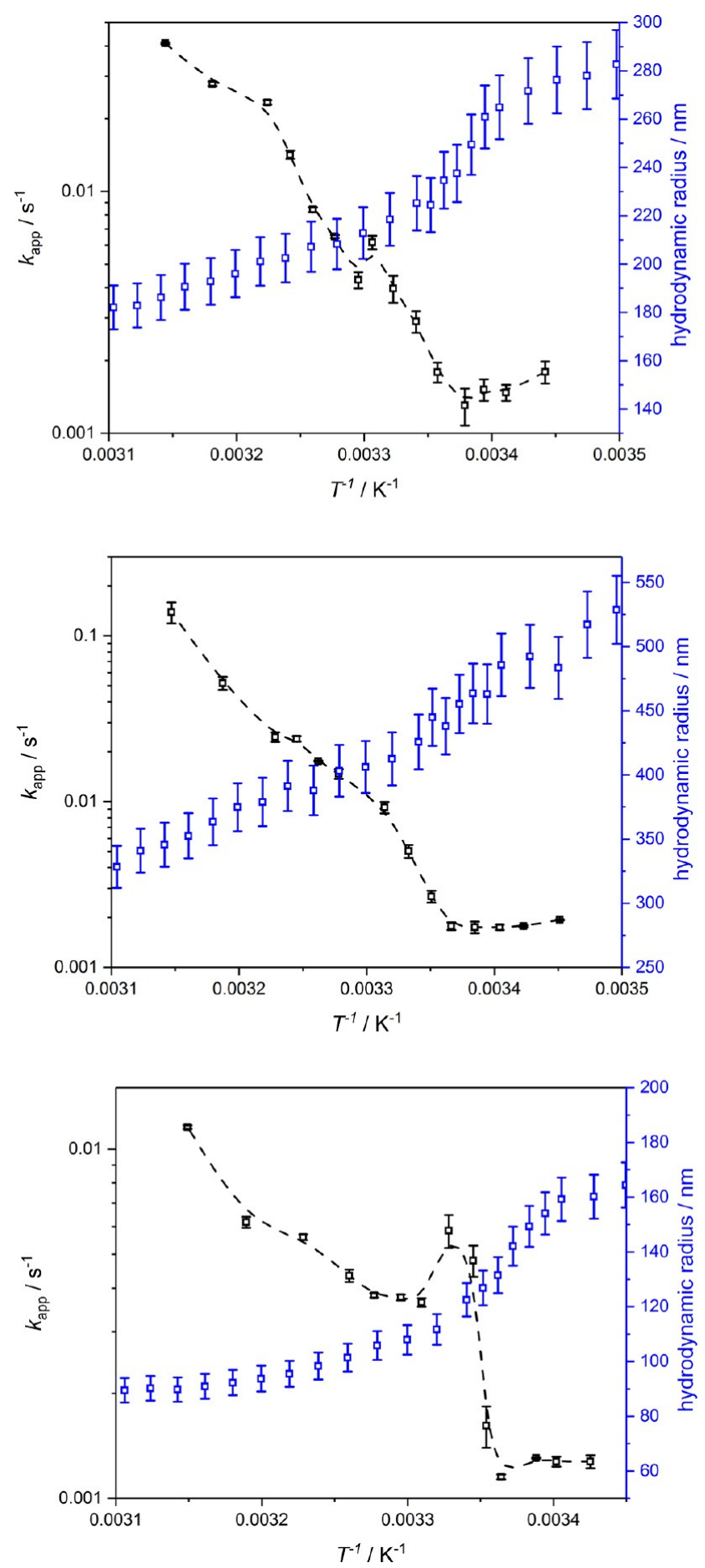

Figure 13. Arrhenius plot of the apparent rate constant of the reduction of $p$-nitrophenole (black squares). The catalytic activity is given for the hybrid systems based on PNI10@PNN (top), PMAM10@PNN (middle), and PNN10@PNN (bottom). For comparison, the swelling curves of the respective core-shell microgels are given (blue squares). The dashed lines serve as guide to the eye.

deviation, which exactly follows the theoretically predicted behavior by Roa et al., ${ }^{47}$ is connected to the VPTT of PNIPAM. However, the observed deviation from the Arrhenius-like behavior is still quite small compared to the core-shell microgels based on polystyrene spheres with a PNIPAM shell, which were used by $\mathrm{Lu}$ et al. ${ }^{50} \mathrm{Lu}$ et al. ${ }^{50}$ observed changes of about 1 or 2 orders of magnitude. The changes in the catalytic activity of our silver nanoparticle hybrids are less pronounced and are in the order of a factor of 5. So the PNIPAM-co-AAc@PNNPAM-based hybrids exhibit a slightly smaller tunability of the catalytic activity compared to the PS@PNIPAM core-shell microgels, but we still observe a deviation from the linear dependency, which is not realized by comparable spherical polyelectrolyte brushes. ${ }^{51,52}$ The general catalytic efficiency of the silver nanoparticles inside the microgel carriers is comparable to other microgel/silver nanoparticle hybrid systems synthesized, for example, by Farooqi et al., ${ }^{27}$ but lower compared to the SPBs published by $\mathrm{Lu}$ et al. ${ }^{51}$ Contrary to the previous systems, the hybrids based on the sample PMAM@PNN obviously do not provide a significant deviation from the Arrhenius-like behavior. We suggest that this is connected to a difference in the mesh size of the copolymer core due to different copolymerization ratios between NIPAM and acrylic acid and NIPMAM and acrylic acid. The NIPMAM-based core-shell microgels seem to exhibit a less dense network, which reduces the capability to hinder the diffusion of the reactants toward the catalysts. The most interesting deviations from the Arrhenius-like behavior are observed for the hybrid system based on the sample PNN10@PNN. Just as expected from the PCS measurements, we observe a completely different course of catalytic activity compared to the other systems. After the typical plateau where the catalytic activity stays very low, a jump in the catalytic activity is observable right after the VPTT of PNNPAM. It seems like the reaction could be switched on and off nearly totally by crossing the VPTT of the system. We suggest that this behavior is connected to the unusual steep phase transition of PNNPAM microgels, which can almost be described as discontinuous. ${ }^{36}$ Possibly the change from the hydrophilic network toward a hydrophobic network increases the concentration of the reactants near the catalysts and therefore allows a strong increase in the catalytic activity. Afterward, we observe a deviation from the Arrhenius-like behavior, which could also be described by the theoretical model developed by Roa et al. ${ }^{47}$ Hence, the change in the mesh size of the coreshell particles also plays an important role here.

To investigate the influence of the cross-linker content of the core, we also measured the catalytic activity of the hybrid systems based on the samples PNI5@PNN, PMAM5@PNN, and PNN5@PNN. The respective curves can be found in Figure 14.

At first, it is to be mentioned that the data for the hybrid microgels shown in Figure 14 exhibit different trends as the systems based on PNI10@PNN and PNN10@PNN, but the deswelling of the carrier shows less influence on the catalytic activity and a comparable trend as the system PMAM10@ PNN. Additionally, the catalytic activity of the hybrid microgels seems to be lower, which is in good agreement with the lower nanoparticle load observed in the TEM measurements. For the sample PNI5@PNN, an increase in the catalytic activity is observed after the collapse of the PNNPAM shell. Afterward, the catalytic activity follows almost the classical Arrhenius-like behavior. The decrease in the catalytic activity, which was observed for the hybrid system based on PNI10@PNN, is not realized for this sample. In contrast to that, the course of the catalytic activity of the hybrid systems based on PMAM5@PNN is very comparable to the sample PMAM10@PNN. The change in the catalytic activity after the VPTT of PNNPAM seems to be stronger than for the other sample, but the lower overall catalytic intensity has to be 

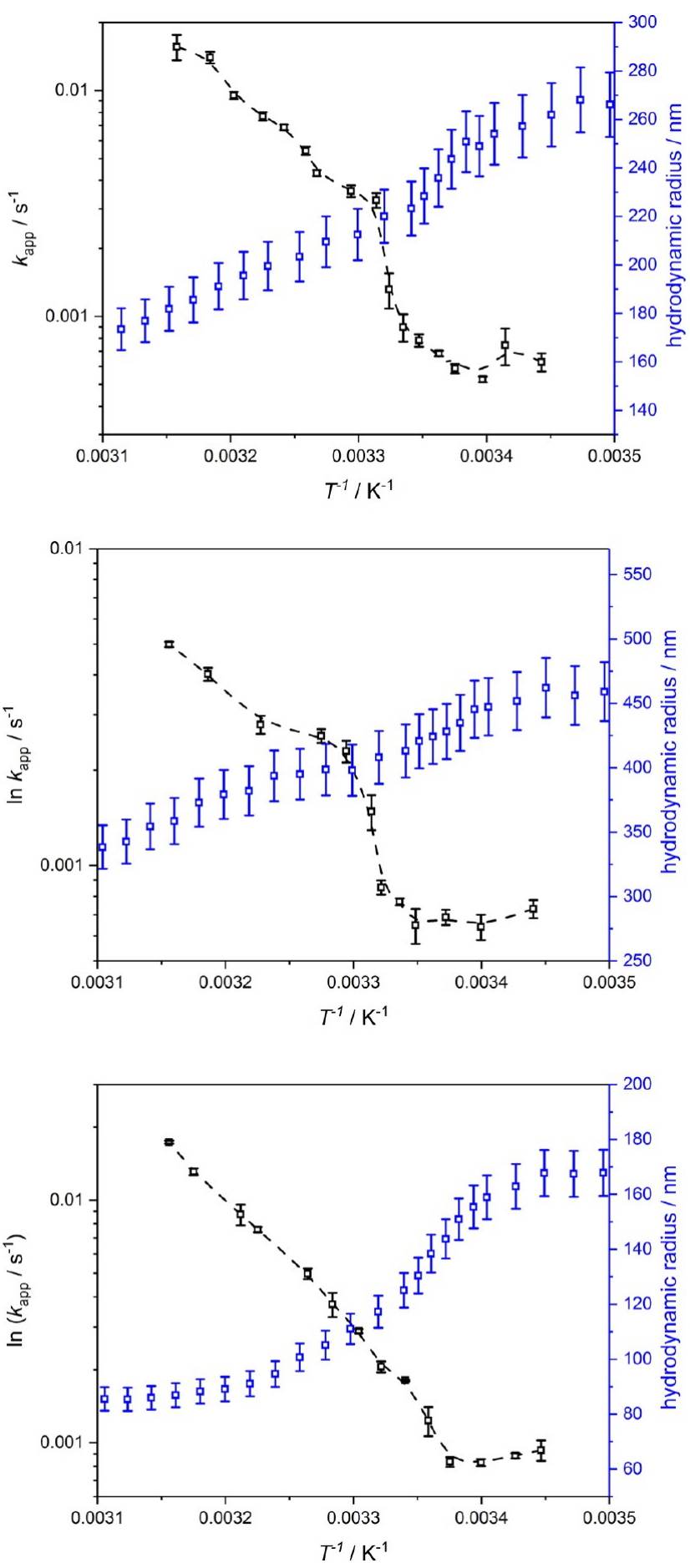

Figure 14. Arrhenius plot of the apparent rate constant of the reduction of $p$-nitrophenole (black squares). The catalytic activities are given for the hybrid systems based on PNI5@PNN (top), PMAM5@PNN (middle), and PNN5@PNN (bottom). For comparison, the swelling curves of the respective core-shell microgels are given (blue squares). The dashed lines serve as guide to the eye.

considered. Therefore, we suppose that the catalytic activity here also almost follows the Arrhenius law. For the hybrid system based on PNN5@PNN, a difference in the course of the catalytic activity occurs when it is compared to the sample with $10 \mathrm{~mol} \%$ cross-linker content in the core. The sample PNN5@PNN shows almost no deviation from the classical Arrhenius-like behavior, so the switchability of the PNNPAM-
co-AAc@PNNPAM microgels seems to disappear in this case. This may be connected to the difference in particle architecture, which was observed in the PCS experiments.

To sum up this part, it can be stated that the cross-linker content of the core is a very important parameter for the switchability of the core-shell microgel/silver nanoparticle hybrids. The cross-linker content of the core shows the strongest influence for the PNNPAM-co-AAc@PNNPAM and PNIPAM-co-AAc@PNNPAM-based materials. In both cases, switchability could only be realized with core-cross-linker contents of $10 \mathrm{~mol} \%$. In the case of PNIPMAM-co-AAc@ PNNPAM carriers, the switchability of the catalytic activity seems to be limited for all cross-linker contents. Interestingly, all microgel/nanoparticle hybrids show a drastic increase of the catalytic activity after the collapse of the PNNPAM shell, which is in good agreement with theoretical descriptions in the literature ${ }^{47,48}$ and could be related to a better solubility of the reactants in the collapsed shell of the core-shell microgels.

\section{CONCLUSIONS}

The present study shows that various acrylamide-based coreshell microgels are suitable carriers for silver nanoparticles. The targeted particle architecture, consisting of a microgel core with silver nanoparticles inside a microgel shell without nanoparticles, could be realized by copolymerization of the microgel core with acrylic acid. TEM measurements and surface plasmon resonance measurements show that the synthesis of silver nanoparticles was successful, and cryoTEM images reveal that the nanoparticles are located mainly at the interface between the core and shell, which can be related to the typical fuzzy structure of acrylic acid containing copolymer microgels. For the nanoparticle stability, the cross-linker density of the core is an important parameter since only microgel templates with a cross-linker-content of $10 \mathrm{~mol} \%$ or lower do not show loss of nanoparticles. By following the reduction of $p$-nitrophenole, it was shown that the volume phase transitions of the shell and the core can influence the catalytic activity of the silver nanoparticles. The fact that different types of monomers like NIPAM, NIPMAM, or NNPAM can be used as the core material increases the temperature range that can be addressed in catalytic processes, although the switchability of the system is influenced by the choice of the core monomer as well. The cross-linker content of the core plays the most important role in the hybrid systems based on PNNPAM-co-AAc@PNNPAM when the catalytic activity is investigated. This seems to be related to a difference in the architecture of the core-shell particles. The use of nonNIPAM-based core-shell microgel/silver nanoparticle hybrid systems is a promising step in the direction toward switchable catalysts.

\section{EXPERIMENTAL SECTION}

4.1. Materials. $N$-isopropylacrylamide (NIPAM) (97\% TCI Germany $\mathrm{GmbH}$, Eschborn, Germany) and $N$-isopropylmethacrylamide (NIPMAM) (97\%, Sigma-Aldrich, Munich, Germany) were recrystallized from $n$-hexane (p.a., VWR International, Darmstadt, Germany). $N, N^{\prime}$-methylenebisacrylamide (BIS) (99\%, Sigma-Aldrich, Munich, Germany), ammonium persulfate (APS) (99\%, Sigma-Aldrich, Munich, Germany), sodium dodecyl sulfate (SDS) (99\%, SigmaAldrich, Munich, Germany), acrylic acid (99\%, Sigma-Aldrich, Munich, Germany), polyethyleneimine (branched, 99\%, 
Sigma-Aldrich, Munich, Germany), ethanol (HPLC grade, VWR International, Darmstadt, Germany), silver nitrate (99.999\%, Sigma-Aldrich, Munich, Germany), 4-nitrophenole (99\%, Carl Roth, Karlsruhe, Germany), and sodium borohydride (98.0\% Sigma-Aldrich, Munich, Germany) were used as obtained. The synthesis of $N$ - $n$-propylacrylamide (NNPAM) was performed via a Schotten-Baumann reaction as described by Hirano et al. ${ }^{53}$ Water was purified and deionized using an Arium pro VF system (Satorius Stedim Systems GmbH, Göttingen, Germany).

4.2. Synthesis of Core-Shell Microgels. A two-step precipitation polymerization following the procedure established by Zeiser et al. ${ }^{38}$ was employed to synthesize all coreshell microgels in this work. First, nine different core microgels were synthesized via the following procedure. The exact amounts of the compounds are presented in Table 2. The

Table 2. Nominal Cross-Linker and Comonomer Amounts for All Synthesized Core Microgels

$\begin{array}{cccc}\text { number } & \text { core monomer } & \text { BIS (mol \%) } & \text { AAc (mol \%) } \\ 1 & \text { NIPAM } & 5 & 5 \\ 2 & \text { NIPAM } & 10 & 5 \\ 3 & \text { NIPAM } & 15 & 5 \\ 4 & \text { NIPMAM } & 5 & 5 \\ 5 & \text { NIPMAM } & 10 & 5 \\ 6 & \text { NIPMAM } & 15 & 5 \\ 7 & \text { NNPAM } & 5 & 5 \\ 8 & \text { NNPAM } & 10 & 5 \\ 9 & \text { NNPAM } & 15 & 5\end{array}$

respective monomer $(11.55 \mathrm{mmol})$ and the cross-linker BIS were dissolved in purified water. The solution was heated up to $70{ }^{\circ} \mathrm{C}$ and purged with nitrogen under constant stirring for 60 min. Acrylic acid (0.58 mmol, $5 \mathrm{~mol} \%)$ and SDS (0.103 $\mathrm{mmol}$ ) were added $10 \mathrm{~min}$ before the initiation. The polymerization was started by adding APS ( $0.41 \mathrm{mmol}, 3.5$ mol \%). The complete reaction volume was $150 \mathrm{~mL}$. The purification was performed by five consecutive centrifugation (25000 rpm, $45 \mathrm{~min}$ ), decantation, and redispersion cycles with purified water.

For the second step of the core-shell synthesis, a dispersion of the respective core microgel $(0.15 \mathrm{wt} \%, 50 \mathrm{~mL})$ was equilibrated with NNPAM $(2.06 \mathrm{mmol})$ and BIS $(0.104 \mathrm{mmol}$, $5 \mathrm{~mol} \%$ with respect to NNPAM) analogous to the core synthesis. Shortly before the initiation with APS $(0.14 \mathrm{mmol}$, $3.5 \mathrm{~mol} \%$ with respect to NNPAM), SDS (0.055 $\mathrm{mmol})$ was added to the solution. The purification process for the coreshell microgels was analogous to the purification of the core microgels. The sample names for the nine resulting core-shell microgels and their exact compositions are presented in Table 1.

4.3. Synthesis of Silver Nanoparticles Inside Microgel Templates. For the synthesis of silver nanoparticles, $50 \mathrm{~mL}$ of a 0.02 wt \% microgel suspension was mixed with $0.28 \mathrm{~mL}$ of a $0.1 \mathrm{M}$ silver nitrate solution. The reaction mixture was cooled with an ice bath under constant nitrogen flux and stirring for 1 h. After that, $5 \mathrm{~mL}$ of a freshly prepared, aqueous sodium borohydride solution $(0.1 \mathrm{M})$ was added slowly to the reaction mixture. The mixture was allowed to reach room temperature directly after the addition of the sodium borohydride. For the purification, $15 \mathrm{~mL}$ of the reaction solution was dialyzed against $5 \mathrm{~L}$ of water for $48 \mathrm{~h}$ to remove the excess of sodium borohydride and the free silver nanoparticles. The water was exchanged two times during the dialysis procedure.

4.4. Atomic Force Microscopy. The sample preparation was performed via spin-coating (rotation speed $1000 \mathrm{rpm}$ ) of $100 \mu \mathrm{L}$ of a 0.05 wt \% microgel solution on a silicon wafer (Siegert Wafer $\mathrm{GmbH}$, Aachen, Germany). Before spincoating, the wafer was successively cleaned with ethanol (HPLC) in a plasma cleaner $\left(\mathrm{O}_{2}\right.$ plasma, Zepto, Diener electronics, Ebhausen, Germany) and coated with a polyethylene imine solution $(0.25 \mathrm{wt} \%)$. The measurements were performed on FlexAFM (Nanosurf GmbH, Langen, Germany) at room temperature in semicontact mode with a cantilever frequency of $200 \mathrm{kHz}$ and a spring constant of $40 \mathrm{~N} / \mathrm{m}$ (Tap300 ALG, Budget Sensors, Innovative Solutions Bulgaria Ltd., Sofia, Bulgaria). The resulting images were analyzed using the free software packages GWYDDION ${ }^{54}$ and Image J. ${ }^{55}$

4.5. Transmission Electron Microscopy. TEM images were taken with a JEOL JEM-2200FS electron microscope (JEOL, Freising, Germany) equipped with a cold field emission electron gun. The microscope was operated at an acceleration voltage of $200 \mathrm{kV}$. For standard room-temperature TEM, carbon-coated copper grids (ECF200-Cu, 200 mesh, Science Services, Munich, Germany) were used. Next, $3 \mu \mathrm{L}$ of a diluted sample solution was dropped on the copper grid, and after $1 \mathrm{~min}$, the residual water was blotted off with a filter paper.

All images were recorded digitally by a bottom-mounted camera (Gatan OneView, Gatan, Pleasanton) and processed with a digital imaging processing system (Digital Micrograph GMS 3, Gatan, Pleasanton). To achieve good statistics, several positions on each grid were imaged. For the analysis of the TEM images, free software Image ${ }^{55}$ was used.

For cryo-TEM, the samples were vitrified on TEM holey carbon grids (Qantifoil R2/1, 200 mesh, Plano GmbH, Wetzlar, Germany) using a Leica blotting and plunging device (Leica EM GP, Leica Mikrosysteme Vertrieb GmbH, Wetzlar, Germany). The grids were plunged into liquid ethane cooled with liquid nitrogen to achieve sufficiently fast cooling. Subsequently, the grids were transferred to a cryo transfer and tomography holder (Fischione Model 2550, E.A. Fischione Instruments, Pittsburgh).

4.6. PCS Measurements. For the particle size determination, all microgel suspensions were highly diluted with a $10 \mathrm{mM}, \mathrm{pH}=7$ buffer solution $\left(c_{\mathrm{MG}}<0.002 \mathrm{wt} \%\right)$. For the investigation of the particle sizes as a function of temperature, a fixed-angle PCS setup with a HeNe-Laser (HNL210L, Thorlabs Inc., Newton, NJ) and an ALV-6010 multiple- $\tau$ correlator (ALV GmbH, Langen, Germany) was used. The scattered light was collected at an angle of $60^{\circ}$ by a singlemode fiber connected to an ALV-detection unit. Temperature control was performed by a thermostated decaline bath. At each temperature, the equilibration time was $25 \mathrm{~min}$, and five measurements were performed. The resulting autocorrelation functions were analyzed via inverse Laplace transformation using CONTIN. ${ }^{56}$ The resulting mean relaxation rates $\bar{\Gamma}$ can be used to calculate the translational diffusion coefficient (see eq 1$)$.

$$
\Gamma=D_{\mathrm{t}} \cdot q^{2}
$$

Here, $q$ is the magnitude of the scattering vector, which can be calculated from the wavelength $\lambda$ of the scattered light, the refractive index $n$ of the sample, and the scattering angle $\theta$. 


$$
q=|\vec{q}|=\frac{4 \pi n}{\lambda} \sin \left(\frac{\theta}{2}\right)
$$

The hydrodynamic radius $R_{\mathrm{h}}$ of the microgel particles can be subsequently computed by using the Stokes-Einstein equation, with the temperature $T$, the Boltzmann constant $k_{\mathrm{b}}$, and the solvent viscosity $\eta$.

$$
D_{\mathrm{t}}=\frac{k_{\mathrm{b}} T}{6 \pi \eta R_{\mathrm{h}}}
$$

4.7. UV-Vis Measurements. The absorption spectra of the silver nanoparticle/microgel hybrid systems were acquired on an Agilent 8453 UV-vis spectrometer (Agilent Technologies Germany, Ratingen, Germany) equipped with a diode array detector and an eight-position sample holder. Temperature was set to $20{ }^{\circ} \mathrm{C}$ and controlled by a thermostat (Haake Phoenix II, Thermo Haake GmbH, Karlsruhe, Germany). The samples were measured in Hellma cuvettes (Hellma GmbH, Mühlheim, Germany). The plasmonic resonance of the silver nanoparticles was measured directly after the purification of the hybrid microgels after 2 weeks and 4 weeks to investigate the aging of the material.

4.8. Measurements of Catalytic Activity. An aqueous mixture of 4-nitrophenole $(0.13 \mathrm{mM}, 0.75 \mathrm{~mL})$ and the respective microgel/silver nanoparticle hybrid (0.02 wt \%, 0.2 $\mathrm{mL}$ ) was prepared and equilibrated at the respective measurement temperature for at least $25 \mathrm{~min}$. The reaction was started by adding aqueous sodium borohydride solution $(100 \mathrm{mM}, 50 \mu \mathrm{L})$. After the addition of sodium borohydride, the absorption spectra of 4-nitrophenole were recorded from 190 to $1100 \mathrm{~nm}$ every $6.4 \mathrm{~s}$ by an Agilent spectrometer (see Section 4.8). Every measurement of kinetics lasted $2400 \mathrm{~s}$. The temperature was controlled by a thermostat (Haake Phoenix II, Thermo Haake GmbH, Karlsruhe, Germany). The temperature of the reaction solution was measured by a PT100temperature sensor. Three measurements were performed for every temperature, and the samples were measured in Hellma cuvettes (Hellma GmbH, Mühlheim, Germany). The analysis of the measured absorption spectra was performed by following the absorption band of 4-nitrophenole at $400 \mathrm{~nm}$. The absorption was analyzed by using a pseudo-first-order approach and to determine the apparent reaction constant $k_{\text {app }}$.

$$
A(t)=A_{0} \exp \left(-k_{\text {app }} \times t\right)
$$

\section{ASSOCIATED CONTENT}

\section{S Supporting Information}

The Supporting Information is available free of charge on the ACS Publications website at DOI: 10.1021/acsomega.8b03511.

PCS relaxation rate distributions of the samples PNI5@ PNN, PMAM5@PNN, and PNN5@PNN; polydispersities of all core-shell templates obtained from fixedangle PCS measurements; exemplaric size distribution of the silver nanoparticles in the sample PNI15@PNN; nanoparticle load and mean radii of the silver nanoparticles in all samples extracted from dry-state TEM measurements (PDF)

\section{AUTHOR INFORMATION}

\section{Corresponding Author}

*E-mail: thomas.hellweg@uni-bielefeld.de.
ORCID

Yvonne Hannappel: 0000-0001-9166-7430

Thomas Hellweg: 0000-0002-2394-5846

Notes

The authors declare no competing financial interest.

\section{ACKNOWLEDGMENTS}

Ina Ehring, Meike Adolph, and Jonna Bookhold are acknowledged for the monomer synthesis. The authors thank the Deutsche Forschungsgemeinschaft (DFG) for financial support (Grants HE2995/6-1 and INST215/444-1). The Nanoanalytic center OWL is acknowledged for the provision of measurement time at the TEM in Bielefeld. We acknowledge support for the Article Processing Charge by the Deutsche Forschungsgemeinschaft and the Open Access Publication Fund of Bielefeld University.

\section{REFERENCES}

(1) Welsch, N.; Ballauff, M.; Lu, Y. In Chemical Design of Responsive Microgels; Pich, A., Albrecht, K., Eds.; Advances in Polymer Science; Springer-Verlag: Berlin, Heidelberg, 2011; Vol. 234; pp 129-163.

(2) Bridot, J.-L.; Faure, A.-C.; Laurent, S.; Rivière, C.; Billotey, C.; Hiba, B.; Janier, M.; Josserand, V.; Coll, J.-L.; Elst, L. V.; Muller, R.; Roux, S.; Perriat, P.; Tillement, O. Hybrid gadolinium oxide nanoparticles: multimodal contrast agents for in vivo imaging. $J$. Am. Chem. Soc. 2007, 129, 5076-5084.

(3) Hifumi, H.; Yamaoka, S.; Tanimoto, A.; Citterio, D.; Suzuki, K. Gadolinium-based hybrid nanoparticles as a positive MR contrast agent. J. Am. Chem. Soc. 2006, 128, 15090-15091.

(4) Kryza, D.; Taleb, J.; Janier, M.; Marmuse, L.; Miladi, I.; Bonazza, P.; Louis, C.; Perriat, P.; Roux, S.; Tillement, O.; Billotey, C. Biodistribution study of nanometric hybrid gadolinium oxide particles as a multimodal SPECT/MR/optical imaging and theragnostic agent. Bioconjugate Chem. 2011, 22, 1145-1152.

(5) Karg, M.; Jaber, S.; Hellweg, T.; Mulvaney, P. Surface plasmon spectroscopy of gold-poly-N-isopropylacrylamide core-shell particles. Langmuir 2011, 27, 820-827.

(6) Dulle, M.; Jaber, S.; Rosenfeldt, S.; Radulescu, A.; Förster, S.; Mulvaney, P.; Karg, M. Plasmonic gold-poly(N-isopropylacrylamide) core-shell colloids with homogeneous density profiles: A small angle scattering study. Phys. Chem. Chem. Phys. 2015, 17, 1354-1367.

(7) Fukushima, D.; Sk, U. H.; Sakamoto, Y.; Nakase, I.; Kojima, C. Dual stimuli-sensitive dendrimers: Photothermogenic gold nanoparticle-loaded thermo-responsive elastin-mimetic dendrimers. Colloids Surf., B 2015, 132, 155-160.

(8) Das, M.; Sanson, N.; Fava, D.; Kumacheva, E. Microgels loaded with gold nanorods: photothermally triggered volume transitions under physiological conditions. Langmuir 2007, 23, 196-201.

(9) Suzuki, D.; Kawaguchi, H. Hybrid microgels with reversibly changeable multiple brilliant color. Langmuir 2006, 22, 3818-3822.

(10) Brugger, B.; Richtering, W. Magnetic, Thermosensitive Microgels as Stimuli-Responsive Emulsifiers Allowing for Remote Control of Separability and Stability of Oil in Water-Emulsions. Adv. Mater. 2007, 19, 2973-2978.

(11) Wang, X.; Shi, Y.; Graff, R. W.; Lee, D.; Gao, H. Developing recyclable $\mathrm{pH}$-responsive magnetic nanoparticles for oil-water separation. Polymer 2015, 72, 361-367.

(12) Schmid, A. J.; Dubbert, J.; Rudov, A. A.; Pedersen, J. S.; Lindner, P.; Karg, M.; Potemkin, I. I.; Richtering, W. Multi-Shell Hollow Nanogels with Responsive Shell Permeability. Sci. Rep. 2016, 6, No. 22736.

(13) Turcu, R.; Craciunescu, I.; Garamus, V. M.; Janko, C.; Lyer, S.; Tietze, R.; Alexiou, C.; Vekas, L. Magnetic microgels for drug targeting applications: Physical-chemical properties and cytotoxicity evaluation. J. Magn. Magn. Mater. 2015, 380, 307-314.

(14) Chai, S.; Zhang, J.; Yang, T.; Yuan, J.; Cheng, S. Thermoresponsive microgel decorated with silica nanoparticles in 
shell: Biomimetic synthesis and drug release application. Colloids Surf., A 2010, 356, 32-39.

(15) Estelrich, J.; Escribano, E.; Queralt, J.; Busquets, M. A. Iron oxide nanoparticles for magnetically-guided and magneticallyresponsive drug delivery. Int. J. Mol. Sci. 2015, 16, 8070-8101.

(16) Kirch, J.; Schneider, A.; Abou, B.; Hopf, A.; Schaefer, U. F.; Schneider, M.; Schall, C.; Wagner, C.; Lehr, C.-M. Optical tweezers reveal relationship between microstructure and nanoparticle penetration of pulmonary mucus. Proc. Natl. Acad. Sci. U. S. A. 2012, 109, 18355-18360.

(17) Pich, A.; Karak, A.; Lu, Y.; Ghosh, A. K.; Adler, H.-J. P. Preparation of Hybrid Microgels Functionalized by Silver Nanoparticles. Macromol. Rapid Commun. 2006, 27, 344-350.

(18) Mei, Y.; Lu, Y.; Polzer, F.; Ballauff, M.; Drechsler, M. Catalytic Activity of Palladium Nanoparticles Encapsulated in Spherical Polyelectrolyte Brushes and Core-Shell Microgels. Chem. Mater. 2007, 19, 1062-1069.

(19) Lu, Y.; Proch, S.; Schrinner, M.; Drechsler, M.; Kempe, R.; Ballauff, M. Thermosensitive core-shell microgel as a "nanoreactor" for catalytic active metal nanoparticles. J. Mater. Chem. 2009, 19, 3955-3961.

(20) Naik, B.; Hazra, S.; Muktesh, P.; Prasad, V. S.; Ghosh, N. N. A Facile Method for Preparation of Ag Nanoparticle Loaded MCM-41 and Study of Its Catalytic Activity for Reduction of 4-Nitrophenol. Sci. Adv. Mater. 2011, 3, 1025-1030.

(21) Tang, Y.; Wu, T.; Hu, B.; Yang, Q.; Liu, L.; Yu, B.; Ding, Y.; Ye, $\mathrm{S}$. Synthesis of thermo- and $\mathrm{pH}$-responsive $\mathrm{Ag}$ nanoparticle-embedded hybrid microgels and their catalytic activity in methylene blue reduction. Mater. Chem. Phys. 2015, 149-150, 460-466.

(22) Jia, H.; Roa, R.; Angioletti-Uberti, S.; Henzler, K.; Ott, A.; Lin, X.; Möser, J.; Kochovski, Z.; Schnegg, A.; Dzubiella, J.; Ballauff, M.; $\mathrm{Lu}, \mathrm{Y}$. Thermosensitive $\mathrm{Cu}_{2} \mathrm{O}-\mathrm{PNIPAM}$ core-shell nanoreactors with tunable photocatalytic activity. J. Mater. Chem. A 2016, 4, 96779684.

(23) Jia, H.; Cao, J.; Lu, Y. Design and fabrication of functional hybrid materials for catalytic applications. Curr. Opin. Green Sustainable Chem. 2017, 4, 16-22.

(24) Satapathy, S. S.; Bhol, P.; Chakkarambath, A.; Mohanta, J.; Samantaray, K.; Bhat, S. K.; Panda, S. K.; Mohanty, P. S.; Si, S. Thermo-responsive PNIPAM-metal hybrids: An efficient nanocatalyst for the reduction of 4-nitrophenol. Appl. Surf. Sci. 2017, 420, 753763.

(25) Khan, S. R.; Farooqi, Z. H.; Ajmal, M.; Siddiq, M.; Khan, A. Synthesis, Characterization, and Silver Nanoparticles Fabrication in $\mathrm{N}$-isopropylacrylamide-Based Polymer Microgels for Rapid Degradation of $p$-Nitrophenol. J. Dispersion Sci. Technol. 2013, 34, 1324-1333.

(26) Farooqi, Z. H.; Siddiq, M. Temperature-Responsive Poly(NIsopropylacrylamide-Acrylamide-Phenylboronic Acid) Microgels for Stabilization of Silver Nanoparticles. J. Dispersion Sci. Technol. 2014, 36, 423-429.

(27) Farooqi, Z. H.; Begum, R.; Naseem, K.; Rubab, U.; Usman, M.; Khan, A.; Ijaz, A. Fabrication of silver nanoparticles in $\mathrm{pH}$ responsive polymer microgel dispersion for catalytic reduction of nitrobenzene in aqueous medium. Russ. J. Phys. Chem. A 2016, 90, 2600-2608.

(28) Thomas, V.; Yallapu, M. M.; Sreedhar, B.; Bajpai, S. K. A versatile strategy to fabricate hydrogel-silver nanocomposites and investigation of their antimicrobial activity. J. Colloid Interface Sci. 2007, 315, 389-395.

(29) Lu, Y.; Ballauff, M. Thermosensitive core-shell microgels: From colloidal model systems to nanoreactors. Prog. Polym. Sci. 2011, 36, 767-792.

(30) Kratz, K.; Eimer, W. Swelling properties of colloidal poly(NIsopropylacrylamide) microgels in solution. Ber. Bunsen-Ges. Phys. Chem. 1998, 102, 848-854.

(31) Pelton, R. Temperature-sensitive aqueous microgels. Adv. Colloid Interface Sci. 2000, 85, 1-33.

(32) Lyon, L. A.; Meng, Z.; Singh, N.; Sorrell, C. D.; St John, A. Thermoresponsive microgel-based materials. Chem. Soc. Rev. 2009, $38,865-874$.
(33) Nayak, S.; Lyon, L. A. Soft Nanotechnology with Soft Nanoparticles. Angew. Chem., Int. Ed. 2005, 44, 7686-7708.

(34) von Nessen, K.; Karg, M.; Hellweg, T. Thermoresponsive poly(N-isopropylmethacrylamide) microgels: Tailoring particle size by interfacial tension control. Polymer 2013, 54, 5499-5510.

(35) Cors, M.; Wrede, O.; Genix, A.-C.; Anselmetti, D.; Oberdisse, J.; Hellweg, T. Core-Shell Microgel-Based Surface Coatings with Linear Thermoresponse. Langmuir 2017, 33, 6804-6811.

(36) Wedel, B.; Hertle, Y.; Wrede, O.; Bookhold, J.; Hellweg, T. Smart Homopolymer Microgels: Influence of the Monomer Structure on the Particle Properties. Polymers 2016, 8, No. 162.

(37) Jones, C. D.; Lyon, L. A. Synthesis and Characterization of Multiresponsive Core-Shell Microgels. Macromolecules 2000, 33, 8301-8306

(38) Zeiser, M.; Freudensprung, I.; Hellweg, T. Linearly thermoresponsive core-shell microgels: Toward a new class of nanoactuators. Polymer 2012, 53, 6096-6101.

(39) Berndt, I.; Pedersen, J. S.; Richtering, W. Temperature-sensitive core-shell microgel particles with dense shell. Angew. Chem., Int. Ed. 2006, 45, 1737-1741.

(40) Karg, M.; Prévost, S.; Brandt, A.; Wallacher, D.; Klitzing, R.; Hellweg, T. In Intelligent Hydrogels; Sadowski, G., Richtering, W., Eds.; Progress in Colloid and Polymer Science; Springer: Cham, 2013; pp 63-76.

(41) Zhang, J.; Xu, S.; Kumacheva, E. Polymer Microgels: Reactors for Semiconductor, Metal, and Magnetic Nanoparticles. J. Am. Chem. Soc. 2004, 126, 7908-7914.

(42) Snowden, M. J.; Chowdhry, B. Z.; Vincent, B.; Morris, G. E. Colloidal copolymer microgels of $\mathrm{N}$-isopropylacrylamide and acrylic acid: $\mathrm{PH}$, ionic strength and temperature effects. J. Chem. Soc., Faraday Trans. 1996, 92, 5013-5016.

(43) Brändel, T.; Wiehemeier, L.; Kottke, T.; Hellweg, T. Microphase separation of smart double-responsive copolymer microgels studied by local fluorescence probes. Polymer 2017, 125, 110116

(44) Wedel, B.; Brändel, T.; Bookhold, J.; Hellweg, T. Role of Anionic Surfactants in the Synthesis of Smart Microgels Based on Different Acrylamides. ACS Omega 2017, 2, 84-90.

(45) Hyatt, J. S.; Douglas, A. M.; Stanley, C.; Do, C.; Barker, T. H.; Fernandez-Nieves, A. Charge segregation in weakly ionized microgels. Phys. Rev. E 2017, 95, No. 012608.

(46) Rehman, S. U.; Siddiq, M.; Al-Lohedan, H.; Sahiner, N. Cationic microgels embedding metal nanoparticles in the reduction of dyes and nitro-phenols. Chem. Eng. J. 2015, 265, 201-209.

(47) Roa, R.; Angioletti-Uberti, S.; Lu, Y.; Dzubiella, J.; Piazza, F.; Ballauff, M. Catalysis by Metallic Nanoparticles in Solution: Thermosensitive Microgels as Nanoreactors. Z. Phys. Chem. 2018, 232, 773-803.

(48) Angioletti-Uberti, S.; Lu, Y.; Ballauff, M.; Dzubiella, J. Theory of Solvation-Controlled Reactions in Stimuli-Responsive Nanoreactors. J. Phys. Chem. C 2015, 119, 15723-15730.

(49) Gu, S.; Wunder, S.; Lu, Y.; Ballauff, M.; Fenger, R.; Rademann, K.; Jaquet, B.; Zaccone, A. Kinetic Analysis of the Catalytic Reduction of 4-Nitrophenol by Metallic Nanoparticles. J. Phys. Chem. C 2014, $118,18618-18625$.

(50) Lu, Y.; Mei, Y.; Drechsler, M.; Ballauff, M. Thermosensitive core-shell particles as carriers for Ag nanoparticles: Modulating the catalytic activity by a phase transition in networks. Angew. Chem., Int. Ed. 2006, 45, 813-816.

(51) Lu, Y.; Mei, Y.; Schrinner, M.; Ballauff, M.; Möller, M. W.; Breu, J. In Situ Formation of Ag Nanoparticles in Spherical Polyacrylic Acid Brushes by UV Irradiation. J. Phys. Chem. C 2007, $111,7676-7681$.

(52) Wunder, S.; Polzer, F.; Lu, Y.; Mei, Y.; Ballauff, M. Kinetic Analysis of Catalytic Reduction of 4-Nitrophenol by Metallic Nanoparticles Immobilized in Spherical Polyelectrolyte Brushes. J. Phys. Chem. C 2010, 114, 8814-8820.

(53) Hirano, T.; Nakamura, K.; Kamikubo, T.; Ishii, S.; Tani, K.; Mori, T.; Sato, T. Hydrogen-bond-assisted syndiotactic-specific <tep- 
common:author-query>AQ6: Please provide a DOI number for ref 53 or indicate if one doesn\&amp; \#x2019;t exist.</tep-common:authorquery $>$ radical polymerizations of $\mathrm{N}$-alkylacrylamides: The effect of the N-substituents on the stereospecificities and unusual large hysteresis in the phase-transition behavior of aqueous solution of syndiotactic poly $(<$ italic $>\mathrm{N}</$ italic $>-<$ italic $>\mathrm{n}</$ italic $>$-propylacrylamide). J. Polym. Sci., Part A: Polym. Chem. 2008, 46, 4575-4583.

(54) Nečas, D.; Klapetek, P. Gwyddion: An open-source software for SPM data analysis. Open Phys. 2012, 10, 181-188.

(55) Rueden, C. T.; Schindelin, J.; Hiner, M. C.; DeZonia, B. E.; Walter, A. E.; Arena, E. T.; Eliceiri, K. W. ImageJ2: ImageJ for the next generation of scientific image data. BMC Bioinf. 2017, 18, No. 529.

(56) Provencher, S. W. CONTIN: A general purpose constrained regularization program for inverting noisy linear algebraic and integral equations. Comput. Phys. Commun. 1982, 27, 229-242. 